\title{
La Représentation du corps dans la littérature, études réunies et présentées par Hamideh LOTFINIA, Mehdi GHASSEMI et Katia HAYEK
}

\section{Luana Doni}

\section{OpenEdition}

\section{Journals}

\section{Edizione digitale}

URL: https://journals.openedition.org/studifrancesi/12296

DOI: 10.4000/studifrancesi. 12296

ISSN: 2427-5856

\section{Editore}

Rosenberg \& Sellier

\section{Edizione cartacea}

Data di pubblicazione: 1 avril 2018

Paginazione: 182-183

ISSN: 0039-2944

\section{Notizia bibliografica digitale}

Luana Doni, «La Représentation du corps dans la littérature, études réunies et présentées par Hamideh LOTFINIA, Mehdi GHaSSEmI et Katia HAYEK», Studi Francesi [Online], 184 (LXII | I) | 2018, online dal 04 juillet 2018, consultato il 17 novembre 2021. URL: http://journals.openedition.org/studifrancesi/12296 ; DOI: https://doi.org/10.4000/studifrancesi.12296

Questo documento è stato generato automaticamente il 17 novembre 2021.

\section{(c) (i) (9)}

Studi Francesi è distribuita con Licenza Creative Commons Attribuzione - Non commerciale - Non opere derivate 4.0 Internazionale. 


\title{
La Représentation du corps dans la littérature, études réunies et présentées par Hamideh LOTFINIA, Mehdi GHASSEMI et Katia HAYEK
}

\author{
Luana Doni
}

\section{NOTIZIA}

La Représentation du corps dans la littérature, études réunies et présentées par Hamideh LOTFINIA, Mehdi GHASSEMI et Katia HAYEK, Lille, Éditions du Conseil Scientifique de l'Université Lille 3, 2016, Collection Travaux et Recherches, 158 pp.

1 Il volume raccoglie gli articoli tratti dagli interventi di ricercatori e dottorandi presentati in occasione della giornata di studi internazionale organizzata alla Maison de la Recherche de l'Université Lille 3, tenutasi il 12 giugno del 2014 e presieduta da Hamideh LOTFINIA, Mehdi GHASSEMI et Katia HAYEK.

2 Il convegno, che aveva per oggetto la rappresentazione del corpo in letteratura, ha permesso ai partecipanti di sviluppare liberamente il tema proposto in ottica interdisciplinare senza restrizioni né temporali né culturali.

Quattro sezioni principali articolano la composizione del volume. La prima sezione dal titolo «Masculin/Féminin» si apre con l'intervento di Alexandre LEROY dal titolo La représentation du corps féminin chez quelques admirateurs célèbres de Rabelais au XIXe siècle (pp. 15-25).

4 Nell'opera di Rabelais il corpo è radicalmente esposto anche nelle sue più basse manifestazioni. Questa singolare maniera di mettere a nudo gli istinti primordiali non può che far risaltare il mutamento, successivo alla morte di Rabelais, nella descrizione 
del corpo ad opera dei suoi più illustri ammiratori. È in particolar modo la descrizione del corpo femminile a presentare vincoli di ordine morale.

Per ovviare al problema della censura di un'opera considerata sconveniente dal punto di vista del costume, l'autore del XIX secolo ricorre all'utilizzo di differenti stereotipi relativi al corpo femminile. Si parla di corpi ma nel contesto di corpi-specifici: Leroy riporta l'esempio di Charles Nodier, il quale tenta di riattualizzare lo stile rabelaisiano ma epurandolo dei contorni più meravigliosi, relegando la presenza del corpo disvelato alla classe popolare. Sono le popolane o le prostitute, a causa della loro mancanza di educazione, a mostrarsi liberamente, senza timori. In Zola, la volgarità di tali soggetti rende la loro identità animalesca, come nel caso di alcuni personaggi de L'Assommoir. In Flaubert invece lo stereotipo femminile presenta frontiere di facile valicabilità. L'esempio di Salambô e di Madame Bovary allontana il mito rabelaisiano del gaudente dalla sfera del basso lignaggio mostrando la possibilità di vedere, se pur in filigrana, un corpo femminile nei suoi istinti più naturali e sensuali.

6 Sono i corpi maschili, nella durezza della loro descrizione, ad essere affrontati nel contributo di Wei si a proposito della scrittrice francese Colette (Le corps masculin chez Colette, pp. 27-39). Nell'opera di Colette, gli uomini sono intermittenti; la strategia operata dall'autrice risulta essere quella della demistificazione dell'eroe tradizionale ottenuta attraverso una rivoluzione dello sguardo che riduce l'uomo a oggetto, passivo e subalterno. La "carne fresca" dei giovani amanti si piega alle volontà della donnasoggetto che non rinuncia ad asservirli ai propri bisogni di natura sensuale. La bellezza dei corpi risiede nella loro giovinezza considerata elemento fondamentale per determinarne l'appetibilità. Lo stesso corpo travestito, quello di Richard conosciuto sotto le sue sembianze femminili con il nome di "Mme Ruby" nel romanzo Bella-Vista, si spiega come il tentativo di opporsi all'avanzare dell'età che pezzo dopo pezzo soffoca la sensualità del corpo. Attraverso il personaggio di Mme Ruby, dalle caratteristiche fortemente virilizzate nonostante le sue apparenze di donna, Colette sovverte i canoni della descrizione tradizionale del corpo e dell'essere maschile, canoni che invece sembrano venir attribuiti alle eroine dei suoi romanzi.

7 C'è chi invece il travestimento lo impiega quale ricorso obbligato di una vita totalmente asservita all'ascetismo come nel caso delle Sante protagoniste del saggio di Joanna AUGUSTYN (Le "no-body" d'une sainte: l'image du corps dans les vies de saintes travesties, pp. 41-48). I corpi descritti dall'A. sono dei non-corpi o, meglio, dei corpi che mirano all'asessualità. Joanna Augustyn riporta l'esempio di Euphrosine che per non contrarre matrimonio fugge di casa in veste d'uomo per raggiungere un'abazia in cui viene introdotta come eunuco. Una dinamica simile la si trova nella vita di Sainte Eugénie che, secondo le parole di Saint Jérôme, rappresenta quella donna che per servire Cristo cessa di essere donna diventando uomo, essendo costui il solo detentore dell'anima. Ciò non basta, proprio nel caso di Eugénie, a tenere lontano il desiderio delle altre donne: vedendosi rifiutate da colei che - travestita - credono uomo, ricorrono all'accusa di stupro costringendola così ingiustamente ad abbandonare i voti.

8 La volontà di privarsi del corpo femminile quale luogo del peccato può spingersi fino alla pratica della mutilazione, come nel caso di Odette e Margaret de Hongrie, considerata risolutiva rispetto a una vita in preda alle minacce del desiderio. Emerge dunque l'atteggiamento ambiguo del rapporto tra le vite delle sante travestite e la loro corporeità: essa è da un lato rinnegata e dall'altro il vero e proprio centro della narrazione. 
9 Il concetto del corpo come oggetto di proprietà fa invece da sfondo all'articolo di Nisrine MALLI (Écriture du corps / corps de l'écriture, pp. 49-61) che presenta le opere di Hanan El-Cheikh e Ghada El-Samman come testimonianze della difficoltà di vivere in una società patriarcale regolata da leggi morali che individuano nella purezza del sesso femminile l'unica forma possibile di dignità. La lotta per la libertà, per il riconoscimento di un'identità culturale e sociale, implica anche quella per la conquista del proprio corpo. Così per Zein e Zahra protagoniste rispettivamente dei romanzi Le roman impossible. Mosaïque damascène e Histoire de Zahara, la libertà sessuale è vista come punto di partenza per la conquista di tutte le altre libertà. La presa di coscienza della frattura nei confronti del passato porta i personaggi alla ridefinizione del concetto di corpo e di relazione mediante la scrittura autobiografica. Il corpo si svela anche nelle sue componenti più intime diventando presenza e autoaffermazione di sé.

10 La seconda sezione della raccolta porta il titolo di «Corps déguisé / corps fragmenté» e si apre con il contributo di Mehdi GHASSEMI (Partial bodies, Ephemeral subjects: uncanny corporeality in John Banville's "Eclipse, Shroud" and "Ancient Light", pp. 65-77) in merito al trattamento del corpo nella trilogia di John Banville.

11 Ciò su cui l'autore riflette è il rapporto tra il narratore e la propria corporeità. Il corpoframmentato di Banville è presentato attraverso un duplice approccio: corpo come sguardo dell'io sul corpo e corpo come sguardo dell'altro sul corpo, le cui componenti vengono analizzate da M. Ghassemi da un punto di vista psicanalitico.

Diverso l'approccio di Luisa MESSINA (La beauté et l'art cosmétique dans le roman libertin du XVIII siècle, pp. 79-88) il cui contributo verte sull'analisi dell'importanza della cura del corpo nella società francese dell'Ancien Régime. La cosmesi in epoca libertina riveste importanza non soltanto da un punto di vista estetico ma anche da un punto di vista sociale. Il viso artefatto assume la funzione di un marchio di appartenenza a una classe sociale, quella della nobiltà frivola e che teme il passare del tempo. L'eccesso nell'uso del trucco è presente nell'opera di Claude Crébillon e ne Les bijoux indiscrets di Denis Diderot come manifestazione ed emblema del brutto e del ridicolo.

13 A concludere la seconda sezione, l'articolo di Maryam ASSADI (Gordafarid, woman of war in male disguise: pushing gender borders through the representation of body, pp. 89-95) incentrato sulla descrizione del personaggio femminile di Gordafarid nello Shahnameh del poeta iraniano Ferdowsi. La giovane eroina di Ferdowsi è costretta a travestirsi da uomo per poter combattere in guerra e sfuggire così ad un ruolo predestinato di moglie e madre.

14 Ad inaugurare la terza sezione della raccolta dal titolo "Corps souffrant», è il contributo di Goran sUBotic (Silence du corps malade dans les mémoires français $d u$ XVII siècle, pp. 99-109) in cui lo studio del corpo malato è articolato su tre livelli. Partendo dal corpo colpito casualmente da malattia, come nel caso del vaiolo e delle sue temibili conseguenze raccontate nei Mémoires di Michel de Merolles, Subotic giunge a quelle malattie considerate come conseguenze di un malessere interiore di cui viene riportato l'esempio nei Mémoires di Brienne le jeune. L'autore conclude con una riflessione sulle "malattie dell'anima" $\mathrm{i}$ cui disagi sono visibili anche esteriormente.

15 I corpi su cui riflette Daniel S. LARANGÉ (Du corpus social au corps individuel. Les figures de l'écriture et de l'empreinte dans "La Saison de l'ombre" de Léonora Miano et "Le Christ selon l'Afrique" de Calixthe Beyala, pp. 111-123) sono legati dai temi comuni della maternità e della vendita del corpo. Se per le donne di Léonora Miano la maternità si trova 
confrontata all'espropriazione e alla ricerca dei propri figli scomparsi nel nulla dopo essere stati rapiti e venduti come schiavi, per la protagonista di Calixthe Beyala è la maternità stessa a identificarsi con la schiavitù del proprio corpo, un corpo costretto nella situazione di madre surrogata alla quale non può scappare a causa di un tacito accordo familiare.

La quarta e ultima sezione del volume, dedicata al "Corps dansant», si apre con l'articolo di Céline tORRENT ( Du corps dansant au poème comme corps. De la danse comme «écriture corporelle» chez Mallarmé à la corporéité de la poésie: une corpoéticité intermédiaire?, pp. 127-139). Attraverso la definizione di "scrittura corporale" data da Mallarmé, Céline Torrent tenta di elaborare il concetto di "corpoeticità". Il corpo nella danza diventa un medium per giungere all'ideale poetico: metamorfizzato dalla danza, il corpo si rivela materia poetica.

Danza e scrittura sono analizzate anche nel contributo di Biliana VASSILEVA (Incarner la poésie dans l'art choréographique contemporain, pp. 141-156) che, prendendo spunto dalle teorie e dalle pratiche di William Forsythe, afferma la necessità di integrare le categorie della danza e del linguaggio verbale. Al di là della semplice rappresentazione, l'immaginario del corpo rinvia a esperienze e sentimenti che il lettore è chiamato a condividere con l'autore e ad allontanarsene allo stesso tempo per elaborare la propria percezione del mondo, lontana da canoni prestabiliti. 